\title{
MUC1 Is Expressed by Human Skin Fibroblasts and Plays a Role in Cell Adhesion and Migration
}

\author{
Priyadarsini Kumar, Jennifer Ji, Twanda L. Thirkill, and Gordon C. Douglas
}

\begin{abstract}
The mucin MUC1 is expressed by normal and cancerous epithelial cells and some nonepithelial cells in which it plays roles in regulating adhesion, migration, and cell signaling. In the present studies we found that MUC1 is expressed by normal human neonatal and adult skin fibroblasts. Fibroblasts are usually considered negative for MUC1 expression. Reverse-transcription polymerase chain reaction and Western blot analyses indicate the presence of full-length MUC1, and immunofluorescence and subcellular fractionation studies show that the protein is expressed on the plasma membrane. Immunohistochemical analyses confirmed the expression of MUC1 by fibroblasts in cryosections of normal human skin. Silencing MUC1 expression in fibroblasts using MUC1 shRNA increased the adhesion of cells to collagen and laminin. Transfection with MUC1 shRNA also increased fibroblast migration on collagen as measured in a wound-healing assay. The expression of $\alpha_{2}$-integrin was increased in MUC1 shRNA-transfected fibroblasts in which it was localized to membrane ruffles, providing a possible explanation for the increased cell migration on collagen. These results extend the range of expression of MUC1 to skin fibroblasts and suggest a functional role for MUC1 in fibroblast adhesion and motility.
\end{abstract}

Key words: adhesion; fibroblasts; integrins; MUC1; skin

\section{Introduction}

$\mathbf{T}$ HE MUCIN MUC1 IS OFTEN acknowledged as having an expression profile that is restricted to normal and cancerous epithelial cells. ${ }^{1}$ However, MUC1 is also expressed by activated $\mathrm{T}$ cells and dendritic cells as well as some nonepithelial cancer cells ${ }^{2-5}$ and embryonic stem cells. ${ }^{6}$ In normal epithelial cells MUC1 is expressed on the apical surface as well as at intracellular locations such as mitochondria and the nucleus. It has multiple functions that include a significant contribution to the mucosal barrier to infection, regulation of cell adhesion and invasion, regulation of metastasis, regulation of cell signaling, and regulation of gene expression through interaction with certain transcription factors/regulators. ${ }^{1,7-11}$ Much of the work relating to our understanding of MUC1 function has been carried out using tumor-derived epithelial cell lines in which MUC1 is highly overexpressed and underglycosylated.

During studies of MUC1 expression in trophoblasts (an epithelial cell type), we unexpectedly observed that MUC1 was expressed by normal human skin fibroblasts that were intended as a negative control. We therefore speculated that MUC1 could be involved in regulating adhesion and migration in these cells. Dermal fibroblasts play important roles in extracellular matrix formation and wound healing, and also have paracrine and autocrine functions. ${ }^{12}$ The experiments described in the present paper characterize the expression of MUC1 in normal human skin fibroblasts using a variety of techniques and demonstrate localization of the protein to the plasma membrane. In other studies we used shRNA knockdown to demonstrate that MUC1 plays a role in fibroblast adhesion to extracellular matrix (ECM) and in migratory activity. Finally, we show that MUC1 appears to regulate the expression of $\alpha_{2}$-integrin, a protein thought to be involved in fibroblast motility.

\section{Methods and Materials}

\section{Antibodies}

The mouse monoclonal antibody DF3 was kindly provided by Fujirebio Diagnostics, Inc. (Malvern, PA). Antibody DF3 was raised against a membrane-enriched fraction of a human breast carcinoma metastatic to $l i v e r^{13}$ and recognizes an epitope within the tandem repeat domain of MUC1. The Armenian hamster monoclonal antibody CT2 $2{ }^{14}$ which recognizes an epitope in the cytoplasmic tail of MUC1, was generously provided by Dr. Sandra Gendler (Mayo Clinic, Arizona). Mouse monoclonal antibody against GAPDH (6C5; sc-32233) was obtained

Department of Cell Biology and Human Anatomy, School of Medicine, University of California, Davis, California. 
from Santa Cruz Biotechnology (Santa Cruz, CA). A rabbit polyclonal antibody against HSP-47 was obtained from Abcam (ab77609, Cambridge, MA). A mouse monoclonal antibody against $\alpha_{2}$-integrin (611016) was obtained from BD Biosciences (San Jose, CA).

\section{Cell culture}

Normal human fibroblasts (NHFs) isolated from human neonatal foreskin and from adult truncal skin were kindly provided by Dr. Rivkah Isseroff (University of California, Davis) ${ }^{15}$ under an approved exemption from the University of California, Davis, Institutional Review Board. Cells were cultured in Dulbecco's modified Eagle's medium (DMEM, low glucose $1 \mathrm{~g} / \mathrm{L}), 10 \%$ fetal bovine serum, and $0.1 \%$ gentamycin. These cells are a mixture of dermal papillary and dermal reticular fibroblasts. Cells were used between passages 5 and 15 . HEK293T cells were cultured in DMEM (high glucose $4 \mathrm{~g}$ / L), $10 \%$ fetal bovine serum, $1 \%$ penicillin/streptomycin with L-glutamine, $1 \%$ sodium pyruvate, and $0.1 \%$ gentamycin.

\section{Immunocytochemistry}

For live immunofluorescence staining, adherent cells on eight-chamber glass LabTek culture slides were blocked in ice-cold phosphate-buffered saline containing $0.2 \%$ gelatin for $1 \mathrm{~h}$ at $4^{\circ} \mathrm{C}$. Cells were then incubated with DF3 primary antibody or mouse IgG1 control antibody (both at $3 \mu \mathrm{g} /$ $\mathrm{mL}$ ) for $1 \mathrm{~h}$ at $4{ }^{\circ} \mathrm{C}$ followed by AlexaFluor-Rhodamine Red-X-labeled goat anti-mouse IgG antibody (Invitrogen Corporation, Carlsbad, CA; diluted 1:400) for $1 \mathrm{~h}$ at $4^{\circ} \mathrm{C}$. Cells were then fixed and permeabilized with ice-cold $3.7 \%$ paraformaldehyde $/ 0.2 \%$ Triton $\mathrm{X}-100$ for $5 \mathrm{~min}$ and washed. The fixed and permeabilized cells were then stained overnight at $4{ }^{\circ} \mathrm{C}$ using antibody against $\mathrm{HSP}-47(1 \mu \mathrm{g} / \mathrm{mL})$. The primary antibody was detected using AlexaFluor-488labeled goat anti-rabbit IgG antibody (Invitrogen; $30 \mathrm{~min}$ at $37^{\circ} \mathrm{C}$ ). Nuclei were stained using $4^{\prime}, 6$-diamidino-2-phenylindole (DAPI). The slides were coverslipped and viewed using a widefield fluorescence microscope. Acquisition settings were kept constant for experimental and control samples. Images were pseudocolorized using Adobe Photoshop (Adobe Systems Incorporated, San Jose, CA).

Staining for $\alpha_{2}$-integrin was carried out on cells fixed and permeabilized with paraformaldehyde and Triton X-100 as already described. The fixed cells were incubated overnight with the mouse monoclonal antibody against $\alpha_{2}$-integrin $(10 \mu \mathrm{g} / \mathrm{mL})$ followed by detection using AlexFluor-488conjugated rabbit anti-mouse $\mathrm{Ig}$.

\section{Immunohistochemistry}

Cryosections $(6 \mu \mathrm{m})$ of normal adult human skin were fixed in $3.7 \%$ formaldehyde for $20 \mathrm{~min}$ and permeabilized in $0.1 \%$ Triton X-100 for $80 \mathrm{~min}$. Sections were then blocked with $10 \%$ normal goat serum for $1 \mathrm{~h}$ and then incubated overnight at $4{ }^{\circ} \mathrm{C}$ with DF3 antibody $(3 \mu \mathrm{g} / \mathrm{mL})$ and anti-HSP-47 antibody $(1 \mu \mathrm{g} / \mathrm{mL})$. After washing, the sections were incubated with secondary antibodies labeled with AlexaFluor-Rhodamine Red X or AlexaFluor-647 for 30 min. DAPI was included to stain nuclei. After further washing, the sections were mounted and viewed with a widefield fluorescence microscope.

\section{Transfection with shRNA}

Lentiviral MUC1shRNA expression plasmid (TRCN0000 122938, SHCLNG-NM_002456) and scrambled shRNA plasmid (pLKO.1-puro Non-Mammalian shRNA Control
FIG. 1. Reverse-transcription polymerase chain reaction (RT-PCR) and Western blot analysis of MUC1 expression. Lysates were prepared from normal human fibroblasts as described in Methods and Materials and assessed by (A) RT-PCR using MUC1-specific primers, (B) Western blot (WB) using DF3 antibody, and (C) native Western blot using DF3 and CT2 antibodies. In other studies, fibroblasts were transfected with MUC1 shRNA or scrambled shRNA and then tested for MUC1 expression using (D) RT-PCR and (E) Western blot. The lane numbers in (A) and (B) correspond to fibroblast lysates from different patients; 1 , neonatal fibroblasts NHF01-0150; 2, neonatal fibroblasts NHF0201; 3, neonatal fibroblasts NHF12-11; 4, adult fibroblasts $12-02$. The images in (B) are a montage of four separate blots.
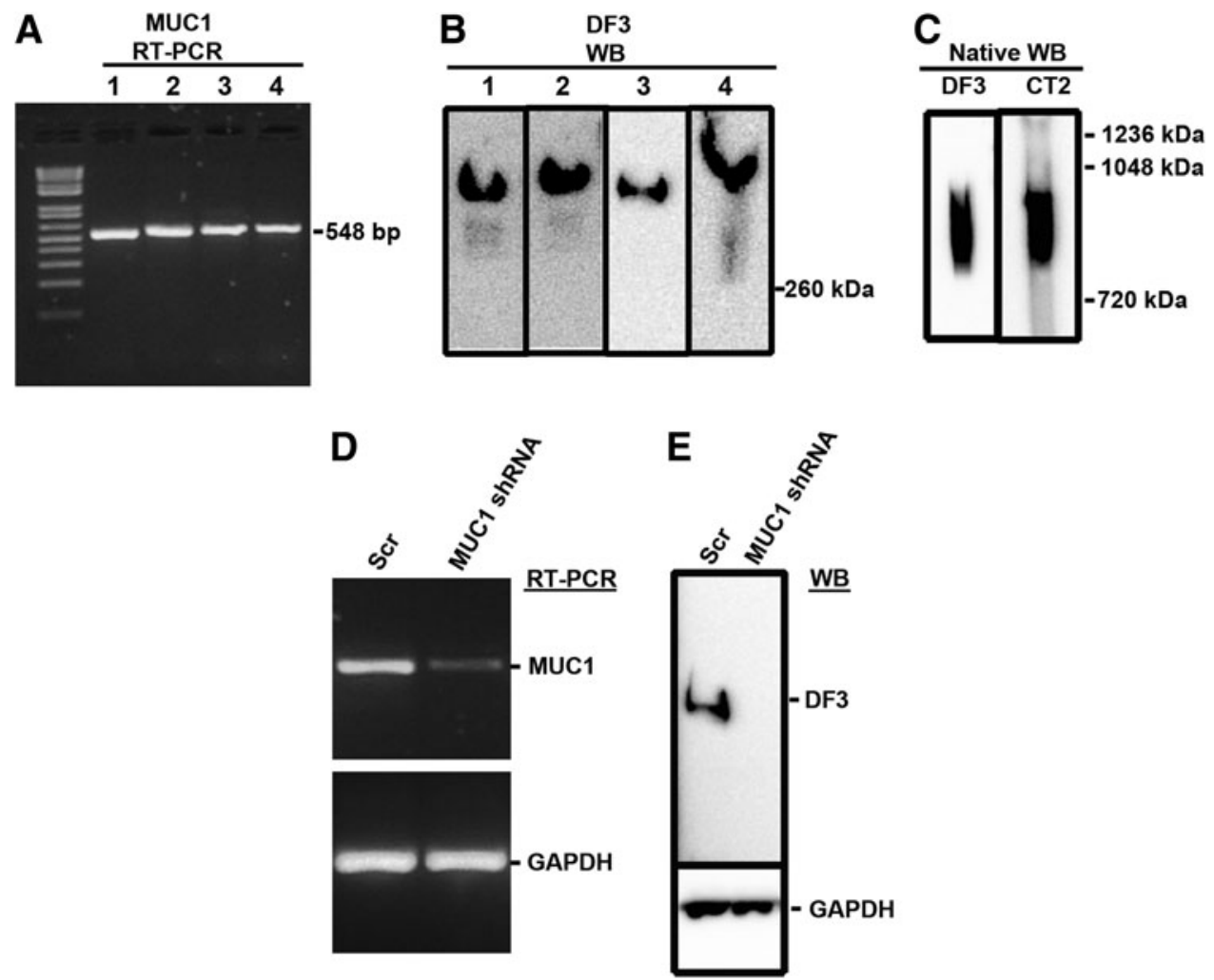
Plasmid DNA SHC002) were purchased from Sigma-Aldrich (St. Louis, MO). HEK293T cells were cotransfected with $10 \mu \mathrm{g}$ of lentiviral plasmids (MUC1 or scrambled), $7.5 \mu \mathrm{g}$ of packaging plasmid, and $5 \mu \mathrm{g}$ of envelope plasmid using Lipofectamine 2000 as the transfection reagent. Culture media were harvested $72 \mathrm{~h}$ post transfection, clarified by centrifugation at $500 \mathrm{~g}$ for $10 \mathrm{~min}$ and stored as aliquots at $-80^{\circ} \mathrm{C}$. NHF cells were transduced with virus particles in the presence of $4 \mu \mathrm{g} / \mathrm{mL}$ polybrene for $5 \mathrm{~h}$ and then selected with $0.85 \mu \mathrm{g} / \mathrm{mL}$ puromycin. Selected cells were maintained in culture medium containing $0.85 \mu \mathrm{g} / \mathrm{mL}$ puromycin.

\section{Western blotting}

Total lysates were obtained by incubating cells in RIPA lysis buffer (Thermo Scientific, Rockford, IL) supplemented with $1 \%$ protease inhibitor cocktail (Sigma-Aldrich) and $1 \mathrm{mM}$ EDTA for $1 \mathrm{~h}$ at $4^{\circ} \mathrm{C}$. Lysates were centrifuged at $13,000 \mathrm{~g}$ for $10 \mathrm{~min}$ at $4^{\circ} \mathrm{C}$ and a protein assay was performed on the supernatant using the BCA assay kit (Thermo Scientific). Fifty micrograms of protein was mixed with NuPAGE LDS sample buffer (Invitrogen) containing dithiothreitol and heated at $70^{\circ} \mathrm{C}$ for $10 \mathrm{~min}$. The samples were centrifuged at $13,000 \mathrm{~g}$ for $2 \mathrm{~min}$ and loaded on 3\%-8\% Tris-Acetate SDSNuPAGE gels (Invitrogen). After electrophoresis the proteins were transferred to polyvinylidene difluoride (PVDF; BioRad, Hercules, CA). The membrane was blocked for $1 \mathrm{~h}$ in $0.5 \%$ casein in Tris-buffered saline plus $0.5 \%$ Tween 20 . For analysis of MUC1 expression, the membrane was incubated overnight with DF3 antibody $(1.2 \mu \mathrm{g} / \mathrm{mL})$, then washed and incubated with secondary antibody labeled with horseradish peroxidase (Thermo Scientific). For analysis of $\alpha_{2}$-integrin,

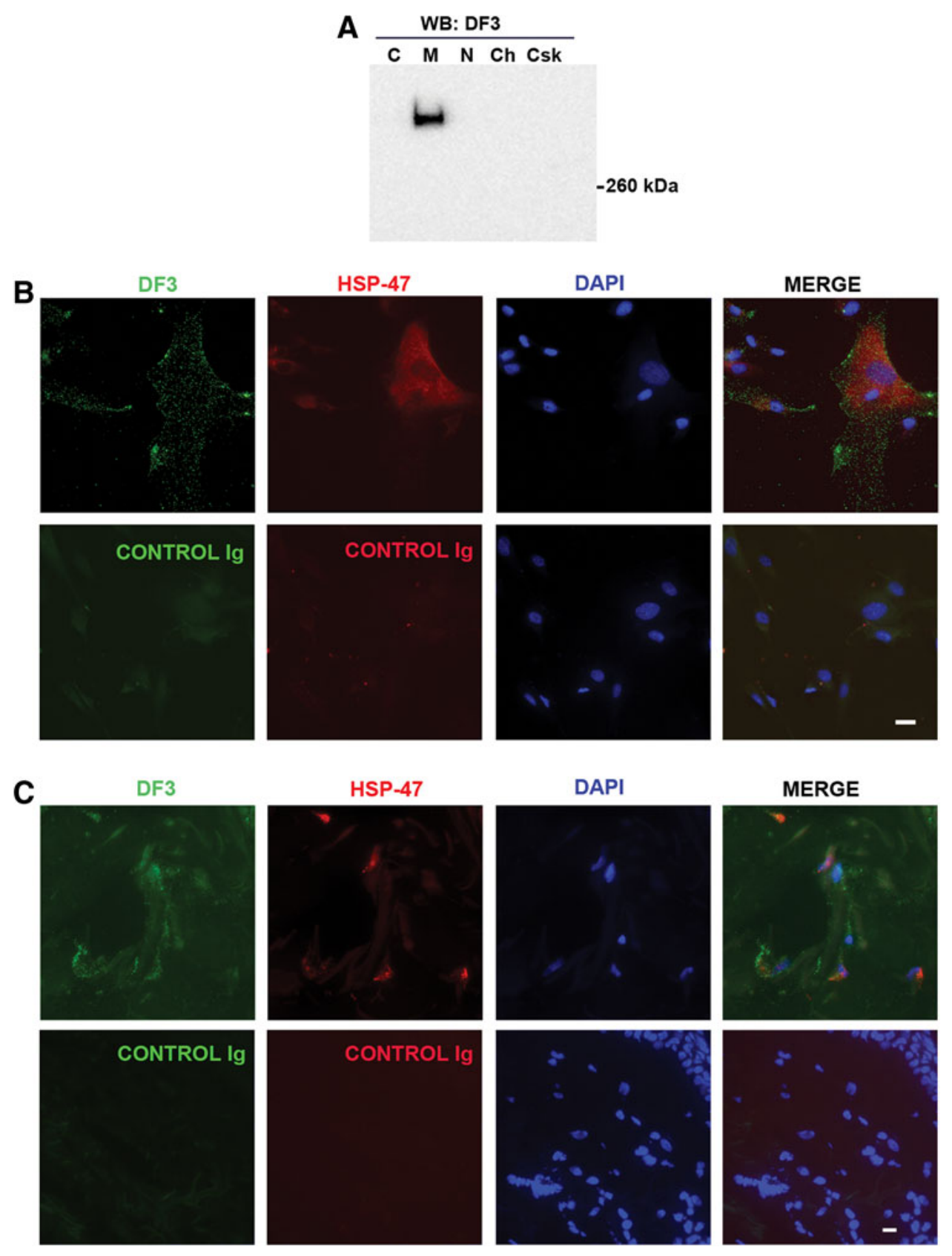

FIG. 2. Subcellular fractionation and immunofluorescence analysis of MUC1 expression. (A) Neonatal fibroblasts were subjected to subcellular fractionation as described in Methods and Materials and individual fractions were then analyzed by Western blot using DF3 antibody. C, cytoplasm; M, membrane; $\mathrm{N}$, nuclear soluble; $\mathrm{Ch}$, chromatin bound; Csk, cytoskeletal. (B) Fibroblasts were chilled to $4^{\circ} \mathrm{C}$ and then live-stained with MUC1 antibody (DF3) or control Ig as described in Methods. After postfixation/permeabilization, cells were stained with antibody against HSP-47 and with DAPI to reveal nuclei. (C) Cryosections of normal adult human skin were stained with MUC1 antibody (DF3) and HSP-47 antibody. The white bars represent $20 \mu \mathrm{m}$. 
membranes were incubated overnight with mouse monoclonal antibody against $\alpha_{2}$-integrin $(0.5 \mu \mathrm{g} / \mathrm{mL})$ followed by incubation with horseradish peroxidase-conjugated secondary antibody. GAPDH was detected using the monoclonal antibody $(0.02 \mu \mathrm{g} / \mathrm{mL})$ described above. After further washing, the membrane was incubated with chemiluminescent substrate (WesternBright Quantum, E\&K Scientific, Santa Clara, CA) and exposed to a Kodak imager (Kodak Imaging Systems, New Haven, CT). Band intensities were analyzed using Kodak Carestream Molecular Imaging Software, and results were normalized to the GAPDH loading control.

\section{Reverse-transcription polymerase chain reaction}

Total RNA was obtained from cells using the RNeasy Plus Mini kit (Qiagen, Valencia, CA) and cDNA synthesized from $1 \mu \mathrm{g}$ of RNA using Superscript II Reverse transcriptase (Invitrogen) was used in polymerase chain reaction (PCR) amplifications using primers shown in Supplementary Table S1. The primers for MUC1 were designed to detect mRNA coding for the full-length species. PCR amplifications were performed using AccuPower PCR premix (Bioneer, Alameda, CA) at an annealing temperature of $60^{\circ} \mathrm{C}$.

\section{Subcellular fractionation}

Subcellular fractionation was carried out using the Subcellular Protein Fractionation Kit (Thermo Scientific) as described by the manufacturer. The procedure yields (1) a cytosolic fraction, (2) a membrane fraction, (3) a nuclear soluble fraction, (4) a nuclear chromatin-bound fraction, and (5) a cytoskeletal fraction. Equal volumes of each fraction were loaded onto the NuPAGE gel and Western blotting was performed as already described.

\section{Native gel electrophoresis}

Protein sample from the membrane fraction isolated as described above was mixed with $2 \times$ native Tris-glycine sample buffer and loaded onto a 3\%-8\% Tris-acetate gel. The gel was run for $3 \mathrm{~h}$ with Tris-glycine native running buffer. The proteins were transferred onto PVDF membrane using NuPAGE transfer buffer plus $10 \%$ methanol, at constant voltage of $30 \mathrm{~V}$ for $20 \mathrm{~h}$. The membrane was blocked, incubated with primary antibodies (DF3 or CT2) overnight, and developed as already described.

\section{Adhesion assay}

Adhesion assay was performed using the ECM Cell Adhesion Array Kit (Colorimetric; EMD Millipore, Billerica, MA). Briefly, NHF cells were prepared as a single cell suspension in assay buffer at a density of $1.5 \times 10^{6}$ cells $/ \mathrm{mL}$. One hundred microliters of the cell suspension was added to the wells and assayed as per the instruction manual. Absorbance at $570 \mathrm{~nm}$ was read on a microplate reader.

\section{In vitro wound healing ("scratch") assay}

NHF cells were cultured in 35-mm dishes (ibiTreat 35-mm dish with 500- $\mu$ m grid; ibidi LLC, Verona, WI) coated with rat tail type 1 collagen and grown to $100 \%$ confluence. Cells were treated with $10 \mu \mathrm{g} / \mathrm{mL}$ mitomycin $\mathrm{C}$ for $1 \mathrm{~h}$ and washed with media. Scratches were made with a $10-\mu \mathrm{L}$ pipette tip and floating cells were removed by washing. $\mathrm{CO}_{2}$-independent medium was added, and cells were incubated in $\mathrm{CO}_{2}$-free $37^{\circ} \mathrm{C}$ incubator until the end of the experiment. Phase
FIG. 3. Effect of MUC1 shRNA on fibroblast adhesion and migration. (A) Neonatal fibroblasts were transfected with MUC1 shRNA or scrambled shRNA, after which adhesion to the indicated matrices was measured as described in Methods. Results are means \pm SEMs. Analysis of the data by two-way ANOVA showed a significant effect of shRNA treatment $\left(F_{1,56}=5.64\right.$, $p=0.021)$ with no significant interaction and no significant effect of matrix. Significant differences $(p<0.05, n=7)$ from the respective scrambled controls are indicated by the asterisks $(*)$. (B) Fibroblasts were transfected with MUC1 shRNA, after which migration on collagen I was assessed using a scratch wound-healing assay as described in Methods. The phase-contrast micrograph shows typical results at zero time and at $6 \mathrm{~h}$. The bar chart shows quantification of migration expressed as \% open wound area. Values are means \pm SEM. The asterisk indicates that the value was significantly different $(p<0.05 ; n=3)$ from the scrambled control.
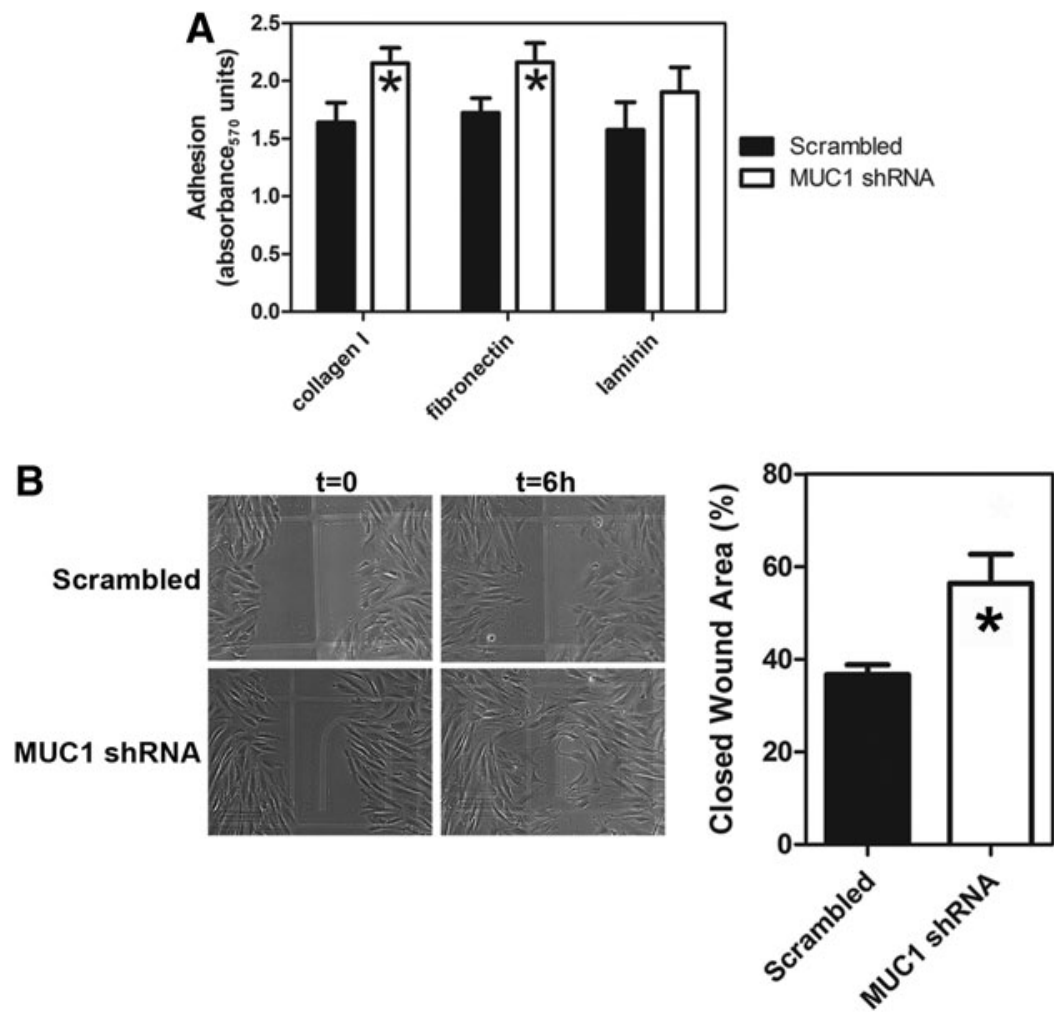
contrast images of scratched areas were taken at desired time points, and the open scratch area was calculated using Tscratch software. ${ }^{16}$

\section{Statistical analyses}

Data were analyzed using Student's $t$-test or two-way ANOVA as appropriate. The analyses were carried out using Prism 5.0 (Graph Pad Software Inc.). Results were considered significant if $\mathrm{p}<0.05$. All experiments were carried out at least three times as indicated in the figure legends.

\section{Results}

\section{Normal human fibroblasts express MUC1 $m R N A$ and protein}

Reverse-transcription (RT)-PCR analysis (Fig. 1A) of neonatal and adult fibroblast lysates using MUC1 primers revealed an amplicon with the expected size and Western blot analysis (Fig. 1B) of lysates using an antibody (DF3) against the VNTR region of MUC1 revealed a protein band of $>260 \mathrm{kDa}$, consistent with MUC1. Western blot analysis after native gel electrophoresis (Fig. 1C) revealed a broad band with very high mass consistent with full length MUC1. Native gel/Western blot analysis using an antibody (CT2) specific for the cytoplasmic tail of MUC1 also detected a broad, high molecular mass product.

Specificity of the expression assays was confirmed by transfecting cells with shRNA directed against MUC1. The results in Fig. 1D and E show that both MUC1 mRNA and MUC1 protein were reduced or absent in cells transfected with MUC1 shRNA compared to cells transfected with the scrambled control.

\section{MUC1 is found in the membrane fraction from fibroblasts}

Western blot analysis of subcellular fractions using the DF3 antibody revealed a band in the membrane fraction (Fig. 2A). This fraction represents the plasma membrane as well as mitochondrial and endoplasmic reticulum/Golgi membranes. No bands were detected in other fractions. When live fibroblast cultures were kept on ice to prevent endocytosis and then stained with an antibody (DF3) against the VNTR region of MUC1, a delicate punctate fluorescence was observed consistent with cell surface expression (Fig. 2B). No punctate fluorescence was observed in cells stained with the control immunoglobulin. The cells also stained positively with an antibody against the fibroblast marker HSP-47 (Fig. 2B). ${ }^{17}$

Next, cryosections of normal adult human skin were stained with the DF3 antibody. The sections were also double-stained with HSP-47 antibody to identify fibroblasts. ${ }^{17}$ HSP-47-positive cells were readily detected and these cells also showed punctate fluorescence using DF3 (Fig. 2C). No DF3 or HSP-47 staining was found in sections stained with control immunoglobulins. As expected, DF3 staining was found in sebaceous and sweat gland cells but these cells were negative for HSP-47 (data not shown).

\section{MUC1 is involved in fibroblast adhesion to ECM}

To test whether MUC1 plays a role in the attachment of fibroblasts to ECM, cells were transfected with MUC1 shRNA or with scrambled shRNA and then allowed to ad- here to different ECM components (Fig. 3A). The extent of adhesion was measured using a colorimetric assay. A twoway ANOVA was used to determine the effects of treatment (MUC1 shRNA versus scramble shRNA) on adhesion. MUC1 shRNA-transfected cells showed significantly increased adhesion to collagen and fibronectin compared to cells treated with scrambled shRNA. Adhesion to laminin showed no significant difference between MUC1-silenced cells and controls.

\section{MUC1 plays a role in fibroblast migration}

A scratch wound-healing assay was used to determine whether MUC1 plays a role in fibroblast migration. Collagen I was used as a physiologically relevant and prototypical substrate. The results (Fig. 3B) showed that there was significantly increased wound closure in cultures transfected with

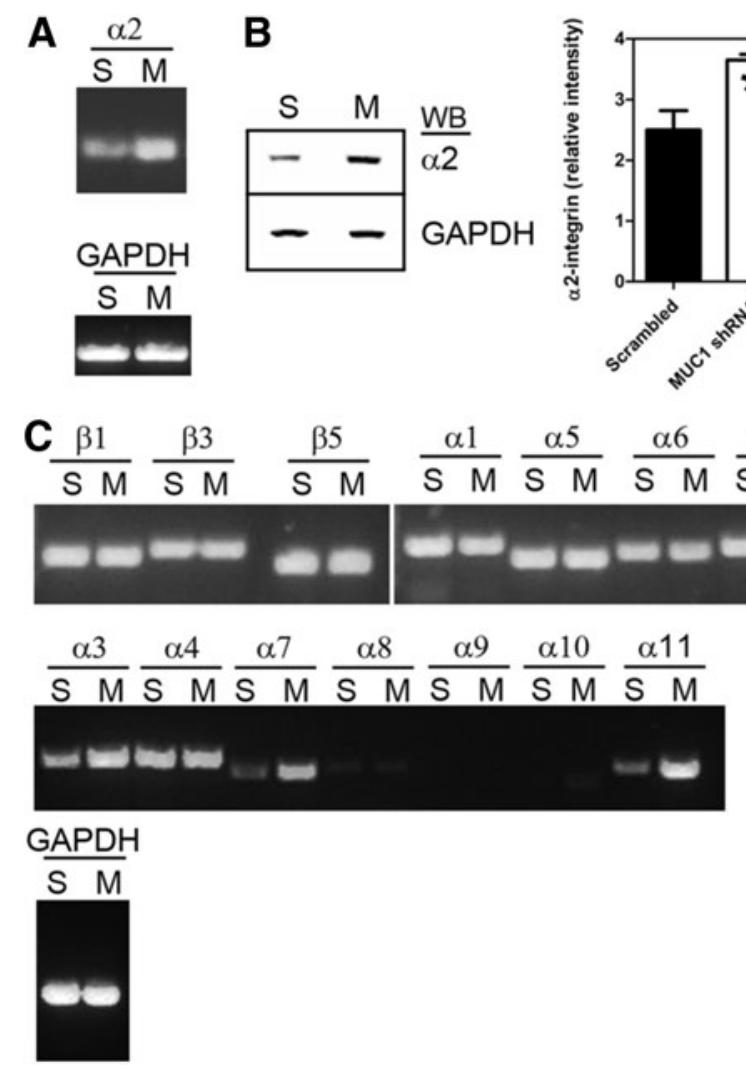

FIG. 4. Effect of MUC1 shRNA on integrin expression by fibroblasts. Neonatal fibroblasts were transfected with MUC1 shRNA (M) or scrambled shRNA (S) as described in Methods. (A) Transfected cells were lysed and analyzed by RT-PCR using primers for $\alpha_{2}$-integrin (see Supplementary Table S1). (B) Transfected cells were lysed and analyzed by Western blotting (WB) using an antibody against $\alpha_{2}$-integrin. GAPDH was used as a loading control. The bar chart shows densitiometric analysis of $\alpha_{2}$-integrin protein bands from three separate experiments. Intensity values (relative to GAPDH) are given as means \pm SEM. The asterisk indicates that the value was significantly different (Student's $t$-test; $p<0.05 ; n=3$ ) from the scrambled control. (C) Transfected cells were analyzed by RT-PCR using a panel of primers against other integrins as indicated (see Supplementary Table S1). 
MUC1 shRNA compared to the control. Since cell proliferation was blocked in this assay, the results are consistent with increased cell migration on collagen when MUC1 is silenced.

\section{MUC1 regulates integrin expression}

We then determined whether expression of $\alpha_{2}$-integrin (an integrin involved in collagen binding) was altered in cells (cultured on collagen) in which MUC1 expression had been silenced. RT-PCR and Western blot analyses of shRNA-transfected cells showed that expression of $\alpha_{2}$ integrin was increased in MUC1 shRNA-transfected cells at both the mRNA (Fig. 4A) and protein levels (Fig. 4B). We also examined the expression of other integrins at the mRNA level (Fig. 4C) and found increased expression of $\alpha_{11}$-integrin but no change in expression of $\alpha_{1}$-integrin (both are involved in collagen binding) in MUC1 shRNAtransfected cells. There was no change in expression of $\beta_{1^{-}}$, $\beta_{3^{-}}, \beta_{5^{-}}, \alpha_{4^{-}}, \alpha_{5^{-}}, \alpha_{6^{-}}$, or $\alpha_{\mathrm{v}}$-integrins compared to control cells, but there was increased expression of $\alpha_{3}$ - and $\alpha_{7}$-integrins (involved in laminin binding).

When $\alpha_{2}$-integrin expression was analyzed using immunofluorescence microscopy, MUC1-silenced cells cultured on collagen showed increased fluorescence in membrane ruffles at cell peripheries compared with cells transfected with scrambled shRNA (Fig. 5).

\section{Discussion}

The studies reported here demonstrate that MUC1 is expressed by normal human skin fibroblasts where it plays a role in the regulation of cell adhesion and migration. To our knowledge these studies represent the first evidence of a functional role for MUC1 in fibroblasts. Indeed, the expression of MUC1 by normal fibroblasts is usually assumed to be absent or insignificant. Based on the RT-PCR results, the MUC1 expressed by human skin fibroblasts is the full-length form. However, further studies will be required to characterize whether other splice variants are expressed. It will also be of interest to examine the expression of $\mathrm{MUC1}{ }^{*}$, a cleaved form of MUC1 found on tumor cells and stem cells. ${ }^{18}$

Skin is sometimes considered to be negative for MUC1 expression. ${ }^{19}$ However, although epithelial cells of the epidermis do not stain with MUC1 antibodies, MUC1 has been detected in sweat gland cells and sebaceous gland cells. ${ }^{20,21}$ MUC1 is also expressed by lymphocytic cells present in normal skin. ${ }^{21}$ In the present studies MUC1 expression was demonstrated in primary cultures of neonatal and adult skin fibroblasts and in fibroblasts within sections of normal adult skin tissue. Fibroblasts were identified using an antibody to HSP-47. ${ }^{17,22,23}$ MUC1 was detected only in the membrane fraction and immunofluorescence analysis of live fibroblasts confirmed cell surface expression of MUC1. MUC1 is expressed on the apical surface of normal epithelial cells, but this polarity is lost in cancer cells. ${ }^{24}$

The observation that silencing MUC1 expression caused increased adhesion of fibroblasts to collagen suggests that normal wild-type levels of MUC1 expression have an antiadhesive role in these cells. This conclusion is similar to the anti-adhesive effects proposed for MUC1 in normal and cancerous epithelial cells. ${ }^{25-28}$ Decreased adhesive ability is thought to promote cell detachment (or prevent attachment) and, in the case of tumor cells, metastasis. Normal fibroblasts are motile within solid tissues ${ }^{29,30}$ and display increased motility during wound healing, ${ }^{31,32}$ and we speculate that expression of MUC1 by skin fibroblasts contributes to this behavior by virtue of its anti-adhesive properties. However, it is more difficult to reconcile this idea with our other observation that silencing of MUC1 increased fibroblast migratory activity (the corollary of which would be that normal expression of MUC1 decreases migration). Conclusions are also confounded by the observation that engineered overexpression of MUC1 in rat fibroblasts increased invasive activity. ${ }^{33}$
FIG. 5. Effect of MUC1 shRNA on $\alpha_{2}$-integrin expression and localization in fibroblasts. Neonatal fibroblasts were transfected with MUC1-shRNA or scrambled shRNA as described in Methods and cultured on collagen I. After $24 \mathrm{~h}$ the cells were fixed, permeabilized, and stained with an antibody against $\alpha_{2}$ integrin. Nuclei were stained with DAPI. An antibody control was included and consisted of incubating cells with an isotype matched Ig in place of the $\alpha_{2}$-integrin antibody. The white arrowhead points to an example of increased fluorescence associated with cell membrane ruffles in the MUC1-silenced cells. The horizontal bar (bottom right) indicates $20 \mu \mathrm{m}$.

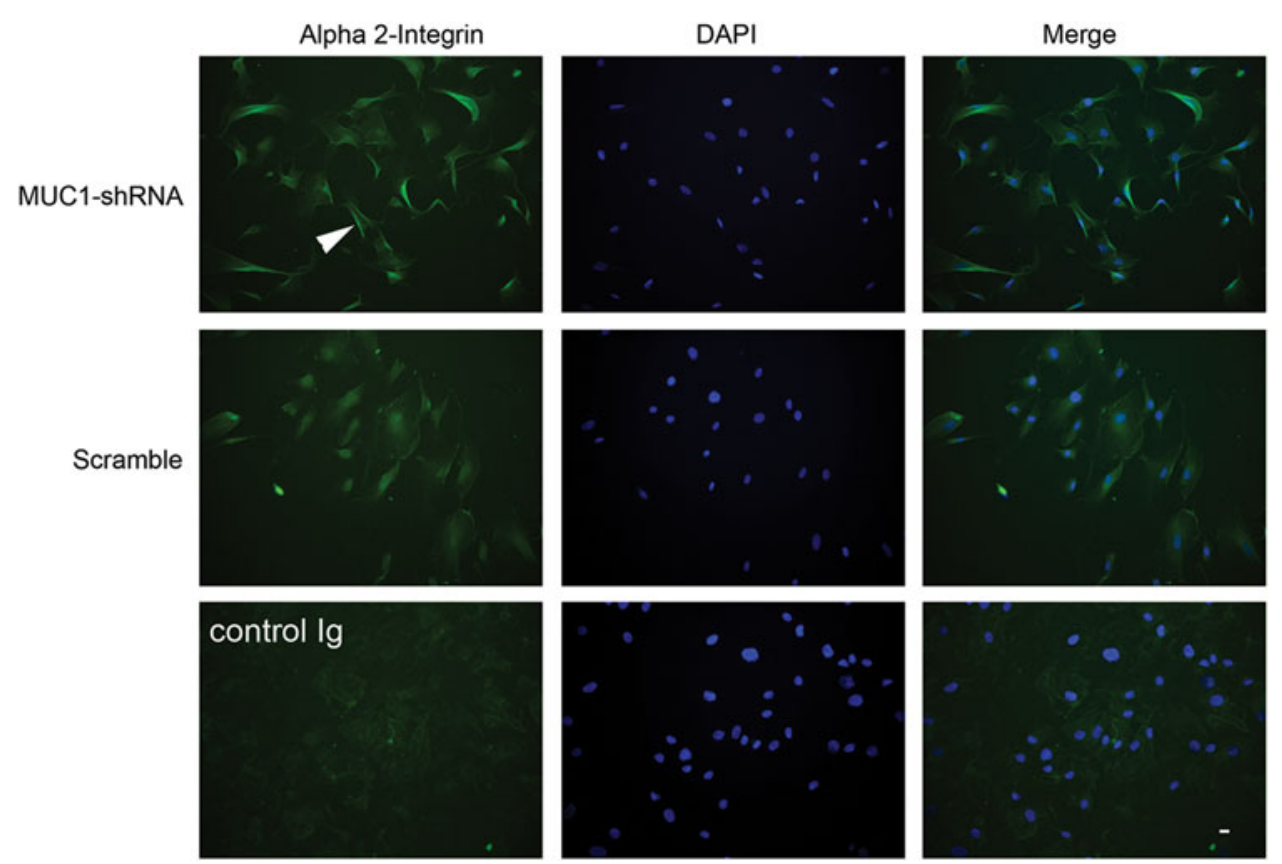


There is much controversy in the literature as to whether MUC1 stimulates or reduces cell adhesion and migration. ${ }^{33-38}$ However, the increased expression of MUC1 found in many carcinomas is generally thought to contribute to increased invasiveness and metastatic potential. ${ }^{11}$ Kohlgraf et al. ${ }^{39}$ found that overexpression of MUC1 caused increased invasion of pancreatic tumor cells in vitro but found decreased invasion when the same cells were implanted in mice. They suggested that MUC1 can function as an antiadhesive or an adhesive molecule depending on interplay between its ability to directly interact with extracellular ligands and its ability to generate intracellular signals that further modulate adhesion and motility. The expression of different MUC1 splice variants by different cell types as well as different overall expression and glycosylation levels could also contribute to the variability in experimental data.

Our finding that $\alpha_{2}$-integrin and $\alpha_{11}$-integrin expression was up-regulated in fibroblasts when MUC1 was silenced provides at least a partial explanation for the increased adhesion and migration observed when cells were cultured on collagen. Expression of $\alpha_{2}$-integrin in the form of the $\alpha_{2} \beta_{1}$ integrin heterodimer confers collagen- and laminin-binding ability, and increased expression of this integrin complex is associated with stimulation of fibroblast migration ${ }^{40,41}$ and with increased adhesion of keratinocytes to collagen. ${ }^{42}$ Alpha 11-integrin also confers collagen-binding activity. ${ }^{43}$ The immunofluorescence studies showed increased expression of $\alpha_{2}$-integrin in cell membrane ruffles in MUC1silenced fibroblasts compared to controls. Membrane ruffles are often found on the cell surface and at the advancing lamellipodium of migrating cells. ${ }^{44}$ In addition to components of the contractile apparatus, integrins are localized to these structures. ${ }^{45}$ The present results are therefore consistent with increased migratory activity in the MUC1-silenced fibroblasts. In contrast to the results for $\alpha_{2}$ - and $\alpha_{11}$-integrins, $\alpha_{1}$-integrin, another collagen-binding integrin, showed no change of expression in MUC1-silenced cells.

Although we restricted our expression studies to the use of collagen as a substrate, MUC1-silenced cells also had increased expression of $\alpha_{3^{-}}$and $\alpha_{7}$-integrins. Although this would be consistent with increased adhesion of the cells to laminin, we found no significant change in adhesion to this substrate. The absence of any observed change in expression of $\alpha_{5}$-integrin (or $\beta_{1}$-integrin) makes it difficult to understand the increased adhesion to fibronectin. One possibility is that MUC1 regulates $\beta_{1}$-integrin activity without changing its expression. The expression of $\alpha_{5^{-}}, \alpha_{\mathrm{v}^{-}}, \alpha_{4^{-}}$, and $\alpha_{2}$-integrin mRNA was not altered when fibroblasts were cultured on fibronectin (results not shown). Further studies are required to uncover the mechanism of the MUC1-dependent upregulation of integrin expression.

In conclusion, the results presented here expand the range of cell types that express MUC1 to include human skin fibroblasts. Further studies will be required to substantiate and define the role of MUC1 in fibroblast motility in vivo and to investigate whether fibroblast MUC1 expression is altered in skin pathologies.

\section{Acknowledgments}

This work was supported by National Institutes of Health Grant 5R01HL068035 (GCD). We thank Drs. Rivkah Isser- off and Marianne Chigbrow (University of California, Davis) for providing cells and tissues.

\section{Author Disclosure Statement}

No competing financial interests exist for any of the authors.

\section{References}

1. Hattrup CL, Gendler SJ. Structure and function of the cell surface (tethered) mucins. Annu Rev Physiol. 2008;70: 431-457.

2. Oosterkamp HM, Scheiner L, Stefanova MC, et al. Comparison of MUC-1 mucin expression in epithelial and nonepithelial cancer cell lines and demonstration of a new short variant form (MUC-1/Z). Int J Cancer. 1997;72:87-94.

3. Vasir B, Avigan D, Wu Z, et al. Dendritic cells induce MUC1 expression and polarization on human T Cells by an IL-7dependent mechanism. J Immunol. 2005;174:2376-2386.

4. Walsh MD, Luckie SM, Cummings MC, et al. Heterogeneity of MUC1 expression by human breast carcinoma cell lines in vivo and in vitro. Breast Cancer Res Treat. 1999; 58:253-264.

5. Kamoshida S, Tsutsumi Y. Expression of MUC-1 glycoprotein in plasma cells, follicular dendritic cells, myofibroblasts and perineurial cells: immunohistochemical analysis using three monoclonal antibodies. Pathol Int. 1998;48:776-785.

6. Hikita ST, Kosik KS, Clegg DO, Bamdad C. MUC1* mediates the growth of human pluripotent stem cells. PLoS One 2008;3:e3312.

7. McAuley JL, Linden SK, Png CW, et al. MUC1 cell surface mucin is a critical element of the mucosal barrier to infection. J Clin Invest. 2007;117:2313-2324.

8. Jonckheere N, Van Seuningen I. The membrane-bound mucins: from cell signalling to transcriptional regulation and expression in epithelial cancers. Biochimie. 2010;92:1-11.

9. Gendler SJ. MUC1, the renaissance molecule. J Mammary Gland Biol Neoplasia. 2001;6:339-353.

10. Roy LD, Sahraei M, Subramani DB, et al. MUC1 enhances invasiveness of pancreatic cancer cells by inducing epithelial to mesenchymal transition. Oncogene. 2011;30:1449-1459.

11. Horm TM, Schroeder JA. MUC1 and metastatic cancer: expression, function and therapeutic targeting. Cell Adh Migr. 2013;7:187-198.

12. Sorrell JM, Caplan AI. Fibroblast heterogeneity: more than skin deep. J Cell Sci. 2004;117:667-675.

13. Kufe D, Inghirami G, Abe M, et al. Differential reactivity of a novel monoclonal antibody (DF3) with human malignant versus benign breast tumors. Hybridoma. 1984;3:223-232.

14. Schroeder JA, Thompson MC, Gardner MM, et al. Transgenic MUC1 interacts with epidermal growth factor receptor and correlates with mitogen-activated protein kinase activation in the mouse mammary gland. J Biol Chem. 2001;276:13057-13064.

15. Sillman AL, Quang DM, Farboud B, et al. Human dermal fibroblasts do not exhibit directional migration on collagen I in direct-current electric fields of physiological strength. Exp Dermatol. 2003;12:396-402.

16. Geback T, Schulz MM, Koumoutsakos P, et al. TScratch: a novel and simple software tool for automated analysis of monolayer wound healing assays. Biotechniques. 2009; 46:265-274.

17. Kuroda K, Tajima S. HSP47 is a useful marker for skin fibroblasts in formalin-fixed, paraffin-embedded tissue specimens. J Cutan Pathol. 2004;31:241-246. 
18. Mahanta S, Fessler SP, Park J, et al. A minimal fragment of MUC1 mediates growth of cancer cells. PLoS One. 2008;3:e2054.

19. Hanisch F-G, Müller S. MUC1: the polymorphic appearance of a human mucin. Glycobiology. 2000;10:439-449.

20. Chakraborty S, Bonthu N, Swanson BJ, et al. Role of mucins in the skin during benign and malignant conditions. Cancer Lett. 2011;301:127-141.

21. Cooper HL, Cook IS, Theaker JM, et al. Expression and glycosylation of MUC1 in epidermolysis bullosa-associated and sporadic cutaneous squamous cell carcinomas. Br J Dermatol. 2004;151:540-545.

22. Kuroda K, Tajima S. Proliferation of HSP47-positive skin fibroblasts in dermatofibroma. J Cutan Pathol. 2008;35: 21-26.

23. Alt E, Yan Y, Gehmert S, et al. Fibroblasts share mesenchymal phenotypes with stem cells, but lack their differentiation and colony-forming potential. Biol Cell. 2011;103:197-208

24. Kufe DW. Mucins in cancer: function, prognosis and therapy. Nat Rev Cancer. 2009;9:874-885.

25. Kondo K, Kohno N, Yokoyama A, et al. Decreased MUC1 expression induces E-cadherin-mediated cell adhesion of breast cancer cell lines. Cancer Res. 1998;58:2014-2019.

26. Wesseling J, van der Valk SW, Vos HL, et al. Episialin (MUC1) overexpression inhibits integrin-mediated cell adhesion to extracellular matrix components. J Cell Biol. 1995;129:255-265.

27. Ligtenberg MJ, Buijs F, Vos HL, et al. Suppression of cellular aggregation by high levels of episialin. Cancer Res. 1992;52:2318-2324.

28. Aplin JD, Meseguer M, Simon C, et al. MUC1, glycans and the cell-surface barrier to embryo implantation. Biochem Soc Trans. 2001;29:153-156.

29. Armstrong MT, Armstrong PB. Cell motility in fibroblast aggregates. J Cell Sci. 1978;33:37-52.

30. Armstrong PB, Armstrong MT. Are cells in solid tissues immobile? Mesonephric mesenchyme studied in vitro. Dev Biol. 1973;35:187-209.

31. Li G, Chen SJ, Oparil S, et al. Direct in vivo evidence demonstrating neointimal migration of adventitial fibroblasts after balloon injury of rat carotid arteries. Circulation. 2000;101:1362-1365.

32. Sandulache VC, Zhou Z, Sherman A, et al. Impact of transplanted fibroblasts on rabbit skin wounds. Arch Otolaryngol Head Neck Surg. 2003;129:345-350.

33. Ye Q, Yan Z, Liao X, et al. MUC1 induces metastasis in esophageal squamous cell carcinoma by upregulating matrix metalloproteinase 13. Lab Invest. 2011;91:778-787.

34. Yuan Z, Wong S, Borrelli A, et al. Down-regulation of MUC1 in cancer cells inhibits cell migration by promoting E-cadherin/catenin complex formation. Biochem Biophys Res Commun. 2007;362:740-746.

35. Costa NR, Paulo P, Caffrey T, et al.. Impact of MUC1 mucin downregulation in the phenotypic characteristics of MKN45 gastric carcinoma cell line. PLoS One. 2011;6:e26970.

36. Bozkaya G, Korhan P, Cokakli M, et al. Cooperative interaction of MUC1 with the $\mathrm{HGF} / \mathrm{c}-$ Met pathway during hepatocarcinogenesis. Mol Cancer. 2012;11:64.
37. Shyu MK, Lin MC, Liu CH, et al. MUC1 expression is increased during human placental development and suppresses trophoblast-like cell invasion in vitro. Biol Reprod. 2008:79:233-239.

38. Rahn JJ, Dabbagh L, Pasdar M, et al. The importance of MUC1 cellular localization in patients with breast carcinoma: an immunohistologic study of 71 patients and review of the literature. Cancer. 2001;91:1973-1982.

39. Kohlgraf KG, Gawron AJ, Higashi M, et al. Contribution of the MUC1 tandem repeat and cytoplasmic tail to invasive and metastatic properties of a pancreatic cancer cell line. Cancer Res. 2003;63:5011-5020.

40. Date H, Furumatsu T, Sakoma Y, et al. GDF-5/7 and bFGF activate integrin alpha2-mediated cellular migration in rabbit ligament fibroblasts. J Orthop Res. 2010;28:225-231.

41. Klein CE, Dressel D, Steinmayer T, et al. Integrin alpha 2 beta 1 is upregulated in fibroblasts and highly aggressive melanoma cells in three-dimensional collagen lattices and mediates the reorganization of collagen I fibrils. J Cell Biol. 1991;115:1427-1436.

42. Chen JD, Kim JP, Zhang K, et al. Epidermal growth factor (EGF) promotes human keratinocyte locomotion on collagen by increasing the alpha 2 integrin subunit. Exp Cell Res. 1993;209:216-223.

43. Velling T, Kusche-Gullberg M, Sejersen T, et al. cDNA cloning and chromosomal localization of human $\alpha_{11}$ integrin: a collagen-binding, i domain-containing, $\beta_{1}$-associated integrin $\alpha$-chain present in muscle tissues. J Biol Chem. 1999;274:25735-25742.

44. Cheresh DA, Leng J, Klemke RL. Regulation of cell contraction and membrane ruffling by distinct signals in migratory cells. J Cell Biol. 1999;146:1107-1116.

45. Conklin MW, Ada-Nguema A, Parsons M, Riching KM, Keely PJ. R-Ras regulates $\beta_{1}$-integrin trafficking via effects on membrane ruffling and endocytosis. BMC Cell Biol. 2010;11:1-15.

Address correspondence to:

Gordon C. Douglas, PhD

Department of Cell Biology and Human Anatomy

School of Medicine

Tupper Hall

One Shields Avenue

University of California Davis

Davis, CA 95616

E-mail: gcdouglas@ucdavis.edu

Abbreviations Used
$\mathrm{DAPI}=4^{\prime}, 6$-diamidino- 2 -phenylindole
$\mathrm{DMEM}=$ Dulbecco's modified Eagle's medium
$\mathrm{ECM}=$ extracellular matrix
$\mathrm{NHF}=$ normal human fibroblast
$\mathrm{PCR}=$ polymerase chain reaction
$\mathrm{PVDF}=$ polyvinylidene difluoride
$\mathrm{RT}-\mathrm{PCR}=$ reverse-transcription PCR

